\title{
The increasing trend in the incidence of gastric cancer in the young population, not only in young Hispanic men
}

\author{
Xing-kang $\mathrm{He}^{1,2} \cdot$ Lei-min Sun ${ }^{1,2}$
}

Received: 23 June 2017 / Accepted: 15 July 2017 / Published online: 24 July 2017

(c) The International Gastric Cancer Association and The Japanese Gastric Cancer Association 2017

Dear Editor,

I read the recent article by Merchant et al. [1] with great interest. They observed an increasing trend in the incidence of gastric cancer in young Hispanic men based on the SEER 13 research dataset between 1992 and 2001. However, when we checked the SEER 18 dataset between 2000 and 2014 using SEER*Explorer, we observed several different outcomes to those noted by Merchant et al. Firstly, according to the results from Merchant et al., the incidence of gastric cancer significantly decreased during the period 2000-2014. However, we found that the APC significantly increased in the young population (20-49 years) between 2000 and 2014 (APC 1.01\%, 95\% CI 0.55-1.47; $P=0.04$ ), whereas Merchant et al. did not observe a significant increase in the APC in the young population. Secondly, in contrast to the results reported by Merchant et al., the APC significantly increased in young Hispanic females (APC $1.80,1.01 \%, 95 \%$ CI $0.69-2.93 ; P<0.01$ ) but not males (APC $-0.05,95 \% \mathrm{CI}-1.21$ to $1.13 ; P=0.93$ ) based on the SEER 18 dataset between 2000 and 2014. We suggest that this inconsistency between our results and those

This comment refers to the article available at doi:10.1007/s10120-016-0603-7.

\footnotetext{
Xing-kang $\mathrm{He}$

hexingkang@zju.edu.cn

Lei-min Sun

sunleimin@yahoo.com

1 Department of Gastroenterology, Sir Run Run Shaw Hospital, Zhejiang University Medical School, Hangzhou 310016, China

2 Institute of Gastroenterology, Zhejiang University (IGZJU), Hangzhou 310016, China
}

of Merchant et al. is due to the different populations investigated in the two studies. The SEER 18 dataset includes data from a considerably larger US population than the SEER 13 dataset does. Therefore, our results are more likely to accurately reflect the recent trend in gastric cancer incidence, and the conclusions of Merchant et al. should be approached with caution. Further studies should be performed to validate the increasing trend in the rate of gastric cancer in the Hispanic population.

\section{Compliance with ethical standards}

Conflict of interest No potential conflicts of interest to declare.

Ethical statement Approval from the ethical board was not required.

\section{Reference}

1. Merchant SJ, Kim J, Choi AH, Sun V, Chao J, Nelson R. A rising trend in the incidence of advanced gastric cancer in young Hispanic men. Gastric Cancer. 2017;20:226-34. 